\title{
Hydrogen-rich saline protects against mitochondrial dysfunction and apoptosis in mice with obstructive jaundice
}

\author{
QU LIU ${ }^{1,2 *}$, BAO-SHAN LI ${ }^{2 *}$, YU-JIAO SONG ${ }^{3 *}$, MING-GEN HU ${ }^{1}$, JIAN-YUE LU ${ }^{2}$, \\ ANG GAO ${ }^{2}$, XUE-JUN SUN ${ }^{4}$, XI-MING GUO ${ }^{3}$ and RONG LIU ${ }^{1}$ \\ ${ }^{1}$ Department of Surgical Oncology, The General Hospital of Chinese People's Liberation Army, Beijing 100853; \\ ${ }^{2}$ Department of General Surgery, People's Liberation Army No. 254 Hospital, Nankai University, Tianjin 300141; \\ ${ }^{3}$ Department of Cell Biology, Beijing Institute of Basic Medical Sciences, Academy of Military Medicine, Beijing 100850; \\ ${ }^{4}$ Department of Diving Medicine, Faculty of Naval Medicine, Second Military Medical University, \\ Shanghai 200433, P.R. China
}

Received March 29, 2015; Accepted January 28, 2016

DOI: $10.3892 / \mathrm{mmr} .2016 .4954$

\begin{abstract}
Previous studies have demonstrated that hydrogen-rich saline (HS) protects against bile duct ligation (BDL)-induced liver injury by suppressing oxidative stress and inflammation. Mitochondria, which are targets of excessive reactive oxygen species and central mediators of apoptosis, have a pivotal role in hepatic injury during obstructive jaundice (OJ); however, the implications of HS in the hepatic mitochondria of BDL mice remain unknown. The present study
\end{abstract}

Correspondence to: Professor Rong Liu, Department of Surgical Oncology, The General Hospital of Chinese People's Liberation Army, 28 Fuxing Road, Beijing 100853, P.R. China

E-mail: liurong52301@163.com

Professor Xi-Ming Guo, Department of Cell Biology, Beijing Institute of Basic Medical Sciences, Academy of Military Medicine, Beijing 100850, P.R. China

E-mail: guoxim@163.com

*Contributed equally

Abbreviations: OJ, obstructive jaundice; ROS, reactive oxygen species; MPT, mitochondrial permeability transition; $\mathrm{H}_{2}$, hydrogen; HS, hydrogen-rich saline; BDL, bile duct ligation; mMDA, mitochondrial malondialdehyde; mGSH, mitochondrial glutathione; mGSSG, mitochondrial glutathione disulfide; mSOD, mitochondrial superoxide dismutase; mCAT, mitochondrial catalase; mGpx, mitochondrial glutathione peroxidase; TUNEL, terminal deoxynucleotidyl transferase dUTP nick end labeling; HBSS, Hank's balanced salt solution; PBS, phosphate-buffered saline; TEM, transmission electron microscopy; SOD2, superoxide dismutase; CsA, cyclosporin A; MPT, mitochondrial potential transition; RCR, respiratory control ratio; $\mathrm{ADP} / \mathrm{O}$, oxidative phosphorylation; ANOVA, analysis of variance; NS, normal saline; MMP, mitochondrial membrane potential

Key words: hydrogen-rich saline, obstructive jaundice, mitochondrial oxidative stress, mitochondrial injury, apoptosis investigated the hypothesis that HS could reduce OJ-induced liver injury through the protection of mitochondrial structure and function, as well as inhibition of the mitochondrial apoptotic pathway. Male C57BL/6 mice were randomly divided into three experimental groups: Sham operation group, BDL injury with normal saline (NS) treatment group, and BDL-injury with HS treatment group. Mitochondrial damage and apoptotic parameters were determined 3 days post-BDL injury and treatment. The results demonstrated that mitochondria isolated from the livers of NS-treated BDL mice exhibited increased mitochondrial swelling, cytochrome $c$ release, and oxidative damage. In addition, liver samples from NS-treated BDL mice exhibited significant increases in B-cell lymphoma 2 (Bcl-2)-associated $\mathrm{X}$ protein expression, caspase activities, and hepatocyte apoptosis compared with livers from sham-operated controls. Notably, treatment with HS reduced the levels of these markers and alleviated morphological defects in the mitochondria following injury. In addition, HS markedly increased the antioxidant potential of mitochondria, as evidenced by elevated adenosine triphosphate levels, mitochondrial respiratory function, and increased levels of active $\mathrm{Bcl}-2$. In conclusion, HS attenuates mitochondrial oxidative stress and dysfunction, and inhibits mitochondrial-mediated apoptosis in the livers of BDL mice.

\section{Introduction}

Obstructive jaundice (OJ) occurs due to occlusion of the common bile duct, or complications associated with surgery, tumor, trauma, gallstones, hepatitis, or idiopathic and metabolic diseases, including primary biliary cirrhosis and sclerosing cholangitis (1). Cholestatic liver injury is accompanied by serious complications, which increase the risk of mortality and morbidity (2). The mechanisms by which cholestasis induce acute liver injury remain controversial; however, intrahepatic accumulation of reactive oxygen species (ROS) is thought to be an important contributory factor (3-5). ROS are able to trigger the opening of mitochondrial permeability transition (MPT) pores in the mitochondrial inner membrane, which nonspecifically transport solutes up to a molecular mass of 
1,500 Da (6). Opening of MPT pores lead to cytochrome $c$ release and apoptosis $(7,8)$. Previous studies have indicated that mitochondrial stress and apoptosis have an important role in hepatic injury in OJ $(8,9)$. In particular, it has been demonstrated that, following cholestasis, an accumulation of bile acids in hepatocytes contributes to cell death and is one of the major pathogenic factors resulting in chronic liver damage and fibrosis (10).

Hydrogen $\left(\mathrm{H}_{2}\right)$, in the gaseous state or dissolved in water, has been reported to have therapeutic value as a selective antioxidant via its ability to reduce cytotoxic ROS and to suppress inflammatory reactions (11-13). Unlike other gaseous molecules, $\mathrm{H}_{2}$ can penetrate the cell membrane to reach subcellular compartments, including mitochondria, which are notoriously difficult to target. The utilization of $\mathrm{H}_{2}$ gas-saturated physiological saline, also known as hydrogen-rich saline (HS), is considered to be less complicated and safer than $\mathrm{H}_{2}$ gas inhalation for clinical application. Furthermore, we have previously demonstrated that HS is able to attenuate bile duct ligation (BDL)-induced liver damage by reducing hepatic oxidative stress and inflammation, and can reduce apoptosis in neonatal brain tissue from a rat model of hypoxia-ischemia $(14,15)$. The present study specifically assessed the impact of BDL-induced injury on the hepatic mitochondria of mice, in order to investigate whether HS was able to exert direct protective effects on the mitochondria, and thus prevent mitochondrial damage and mitochondria-induced hepatocyte apoptosis.

\section{Materials and methods}

HS production. HS was prepared as previously described (16). HS was freshly prepared on a weekly basis to ensure that a concentration $>0.6 \mathrm{mmol} / \mathrm{l}$ was maintained.

Experimental protocol. Male C57BL/6 mice, weighing 22-25 g, were obtained from the Experimental Animal Center of Chinese Academy of Sciences (Shanghai, China). Mice received ad libitum access to standard rodent chow and tap water, and were maintained under a natural day/night cycle. All experimental procedures were approved by the Institutional Animal Care and Use Committee of Nankai University (Tianjin, China).

Mice were randomly divided into three experimental groups, each containing 20 mice. Group 1 animals underwent a sham operation and were treated with normal saline (NS; $10 \mathrm{ml} / \mathrm{kg}$ ); group 2 animals underwent BDL and were treated with NS (10 ml/kg); and group 3 animals underwent BDL and were treated with HS $(10 \mathrm{ml} / \mathrm{kg})$.

Prior to the operation, mice were fasted for $12 \mathrm{~h}$ with ad libitum access to water. Each mouse was weighed and anesthetized with pentobarbital $(50 \mathrm{mg} / \mathrm{kg}$; i.p.; Shanghai Reagent Factory, Shanghai, China). Following a midline incision, the common bile duct was exposed and a double-ligature was performed using 6-0 silk suture, causing the bile duct to be sectioned between the ligatures. In sham-operated animals, the common bile duct was freed from the surrounding soft tissue without ligation. A running suture was used for abdominal closure using 2-0 nylon. NS or HS was administered intraperitoneally at 14:00 every day, beginning $2 \mathrm{~h}$ prior to the operation and continuing until 2 days after. Mice were sacrificed via cervical dislocation according to protocol 3 days after BDL.

Preparation of mitochondrial and cytosolic fractions. Following homogenization of the liver tissues, liver mitochondria and cytosol were prepared by differential centrifugation using a mitochondria isolation kit (Pierce Biotechnology, Inc., Rockford, IL, USA), according to manufacturer's protocol. The resulting supernatant contained soluble mitochondrial protein, which was used for western blot analysis of B-cell lymphoma 2 (Bcl-2), Bcl-2-associated X protein (Bax), and mitochondrial cytochrome $c$. Protein content was determined using a bicinchoninic acid (BCA) protein assay kit (Pierce Biotechnology, Inc.).

Determination of antioxidant and lipid peroxidation levels in mitochondria. The mitochondrial suspension was acidified with 2\% 3-[(3-cholamidopropyl)dimethylammonio]-1-propanesulfonate in Tris-buffered saline (TBS) and centrifuged at $9,055.8 \mathrm{x} \mathrm{g}$ for $2 \mathrm{~min}$ at room temperature, according to manufacturer's protocol for the mitochondria isolation kit. The supernatant was analyzed for mitochondrial malondialdehyde (mMDA), mitochondrial glutathione (mGSH), mitochondrial glutathione disulfide (mGSSG), mitochondrial superoxide dismutase (mSOD), mitochondrial catalase (mCAT), and mitochondrial glutathione peroxidase $(\mathrm{mGpx})$ levels. mMDA levels, and reduced and oxidized $\mathrm{mGSH}$ levels were assessed spectrophotometrically, according to previously described methods $(16,17)$. mSOD, mCAT and mGpx activities were assessed using commercial enzyme-linked immunosorbent assay kits, according to the manufacturer's protocols (Nanjing Jiangcheng Bioengineering Institute, Nanjing, China). All readings were taken using a spectrophotometer (Synergy 2; BioTek Instruments, Inc., Winooski, VT, USA). All assays were conducted in duplicate. Protein content in each sample was determined using a BCA protein assay kit (Pierce Biotechnology, Inc.).

Terminal deoxynucleotidyl transferase dUTP nick end labeling (TUNEL) staining. Following dehydration, permeation and methanol fixation, TUNEL staining was performed on 5- $\mu$ m-thick paraffin-embedded sections using an In Situ Cell Death Detection kit (Nanjing Keygen Biotech. Co. Ltd., Nanjing, China), according to manufacturer's protocol, in order to detect apoptotic hepatocytes. TUNEL-positive cells were detected using an Olympus IX70 fluorescence microscope (Olympus Corporation, Tokyo, Japan).

Fluorescence-activatedcellsorting(FACS) analysis.Single-cell suspensions were prepared using a Tissue Dissociation kit (Nanjing Keygen Biotech. Co. Ltd.), according to the manufacturer's protocol. Hepatocyte apoptosis was measured by flow cytometric analysis using an Annexin V-fluorescein isothiocyanate (FITC) assay (Nanjing Keygen Biotech. Co. Ltd.), according to the manufacturer's protocol. Hepatocytes were stained with Annexin V and propidium iodide (PI; BD Biosciences, San Diego, CA, USA) (18) and apoptotic cells were identified as Annexin V-positive/PI-negative. Analysis was performed using the BD FACSAria flow cytometer (BD Biosciences). 

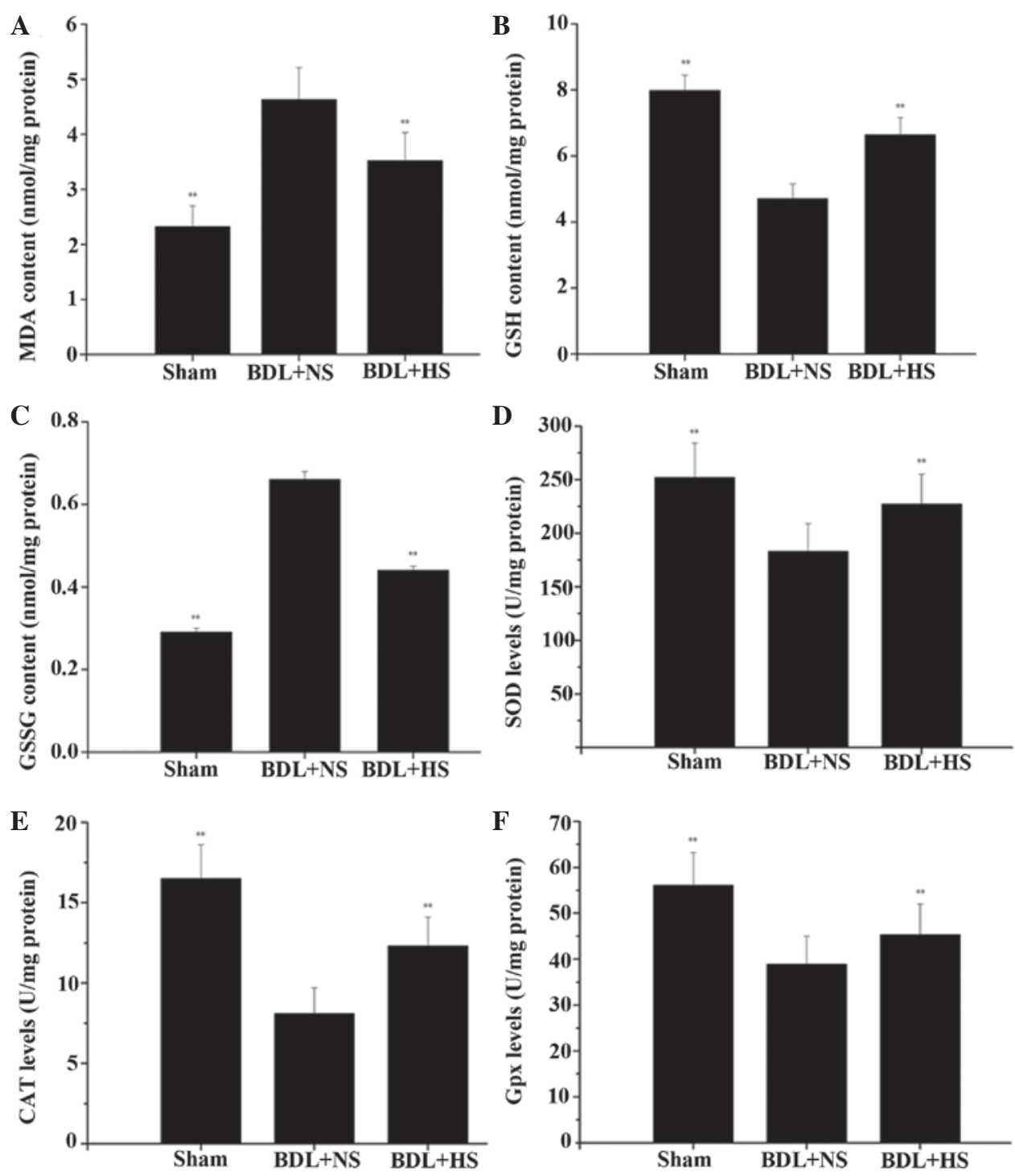

Figure 1. Effects of HS on liver mitochondrial enzyme levels and activities following BDL in mice. (A) Mitochondrial MDA levels; (B) mitochondrial GSH levels; (C) mitochondrial GSSG levels; (D) mitochondrial SOD activities; (E) mitochondrial CAT activities and (F) mitochondrial Gpx activities were measured spectrophotometrically, according to the manufacturers' protocols. Data are presented as the mean \pm standard deviation. ${ }^{* * *} \mathrm{P}<0.01 \mathrm{vs}$. the BDL $+\mathrm{NS}$ group. HS, hydrogen-rich saline; BDL, bile duct ligation; NS, normal saline; MDA, malondialdehyde; GSH, glutathione; GSSG, glutathione disulfide; SOD, superoxide dismutase; CAT, catalase; Gpx, glutathione peroxidase.

Caspase activity assay. Caspase 3,8 and 9 activities were measured in liver tissue using Caspase Assay kits (Promega Corporation, Madison, WI, USA), according to the manufacturer's protocols. The luminescence of each sample was measured using a spectrophotometer (Synergy 2; BioTek Instruments, Inc.).

Mitochondrial swelling. Fresh liver mitochondria were isolated from the BDL and sham-operated mice by differential centrifugation and incubated with $100 \mu \mathrm{M} \mathrm{CaCl}_{2}$ prior to treatment with cyclosporin A (CsA, $1 \mathrm{mM}$; Amresco, LLC, Solon, OH, USA) MPT inhibitor to assess calcium-induced mitochondrial swelling, as previously described (19).

Western blot analysis. Mitochondrial lysates were used for western blot analysis of cytochrome $c, \mathrm{Bcl}-2$ and Bax. Cytosolic fractions were used for western blot analysis of cytochrome $c$, and were processed according to the manufacturer's protocol
(Abcam, Cambridge, MA, USA). Proteins $(2.5 \mu \mathrm{g} / \mu \mathrm{l})$ were separated by $10 \%$ sodium dodecyl sulfate-polyacrylamide gel electrophoresis and transferred onto a polyvinylidene difluoride membrane (Bio-Rad Laboratories, Hercules, CA, USA). Following blocking with 5\% skimmed milk, the membranes were washed three times in TBS with Tween 20 (TBST) for $5 \mathrm{~min}$, and subsequently incubated with primary monoclonal antibodies against cytochrome $c$ (ab13575), Bcl-2 (ab32503) and Bax (all 1:1,000; ab117115) for $2 \mathrm{~h}$ at room temperature. Following washing three times with TBST for $5 \mathrm{~min}$, the membranes were subsequently incubated with goat anti-mouse and rabbit secondary antibodies (1:2,000; DC02L-200UG; Calbiochem, LaJolla, CA, USA) for $2 \mathrm{~h}$ at room temperature. For all determinations, mouse monoclonal anti-glyceraldehyde 3-phosphate dehydrogenase antibody (1:5,000; ab9485; Abcam)) was used as a loading control and goat anti-manganese superoxide dismutase antibody (1:1,000; 13194; Cell Signaling Technology, Inc., Danvers, MA, USA) 
$\mathbf{A}$

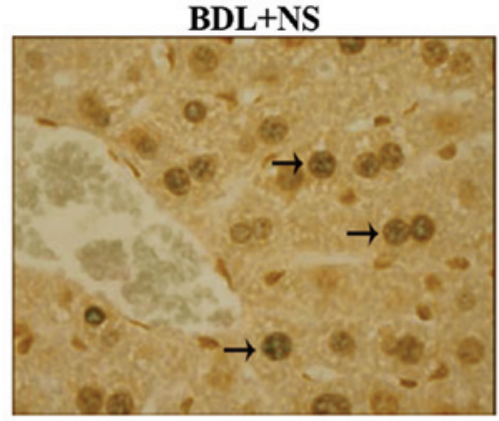

$\mathbf{B}$

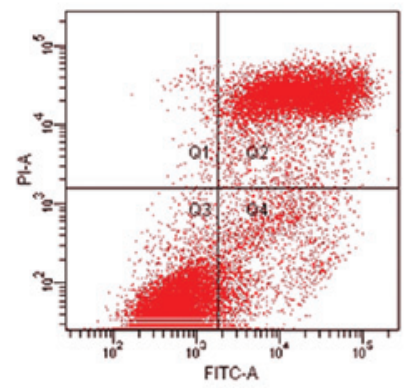

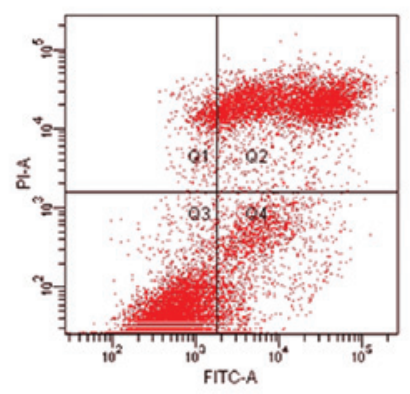

BDL+HS

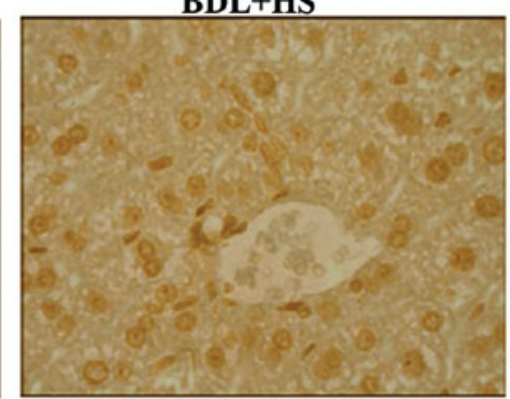

C

D
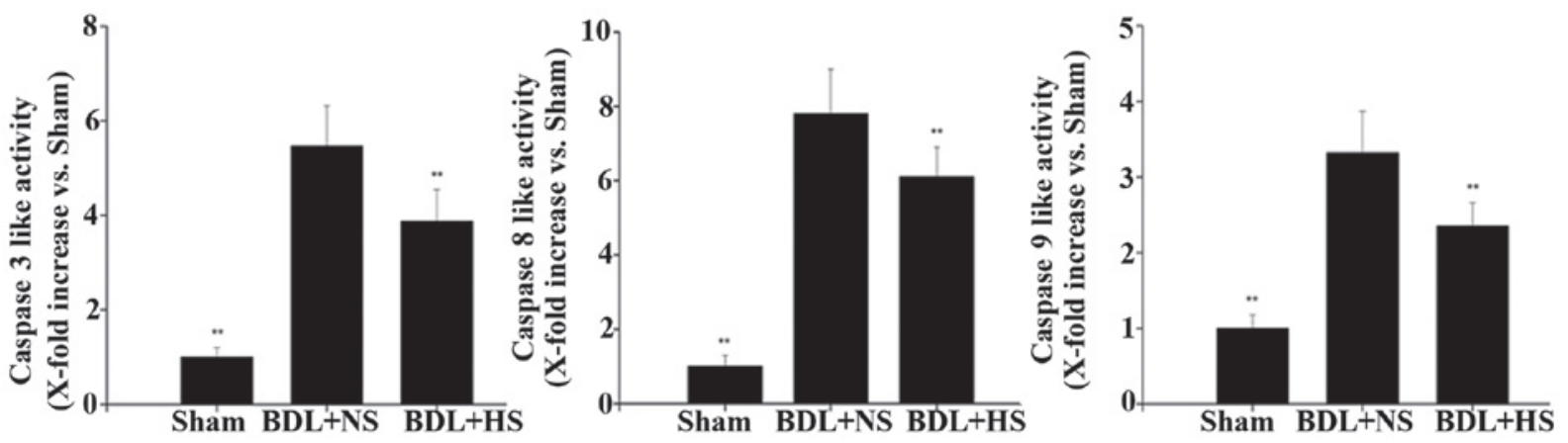

Figure 2. HS treatment decreases hepatocyte apoptosis in BDL mice. (A) Liver samples were sectioned and stained with TUNEL. Notably, HS markedly reduced the number of TUNEL-positive cells in HS-treated livers compared with NS-treated livers (magnification, 100x). Arrows indicate TUNEL-positive cells. (B) Apoptosis was assessed by flow cytometric analysis after the hepatocytes were stained with Annexin V-FITC/PI. (C) Quantitative analysis of the percentage of apoptotic cells. Data of three replicates are presented as the mean \pm standard deviation. ${ }^{* * *} \mathrm{P}<0.01$ vs. the BDL + NS group. (D) Caspase 3,8 and 9 activities were measured by colorimetric assays with a spectrophotometer. Data are presented as the mean \pm standard deviation. HS, hydrogen-rich saline; BDL, bile duct ligation; NS, normal saline; TUNEL, Terminal deoxynucleotidyl transferase dUTP nick end labeling; FITC, fluorescein isothiocyanate; PI, propidium iodide.

was used as a mitochondrial loading control. Blots were visualized using a Beyotime enhanced chemiluminescence Plus substrate system (Beyotime Institute of Biotechnology, Haimen, China) and analyzed with Quantity One 4.62 software (Bio-Rad Laboratories). It should be noted that it has previously been suggested that cytoplasmic cytochrome $c$ may exist in polymeric forms, appearing as a $58-60 \mathrm{kD}$ protein-sized band in western blots, rather than in monomeric form, which migrates at a reduced molecular weight of $15 \mathrm{kD}(20)$.

Transmission electron microscopy (TEM). Liver samples were fixed in a $2 \%$ solution of glutaraldehyde and post-fixed in osmium tetroxide, prior to embedding in epoxy resin for TEM. Ultrathin sections $(40-50 \mathrm{~nm})$ were stained with uranyl acetate and lead citrate, and were then examined under a TEM (Hitachi H-7650; Hitachi, Tokyo, Japan).

Determination of mitochondrial adenosine triphosphate (ATP) content. ATP content was measured using the ATP

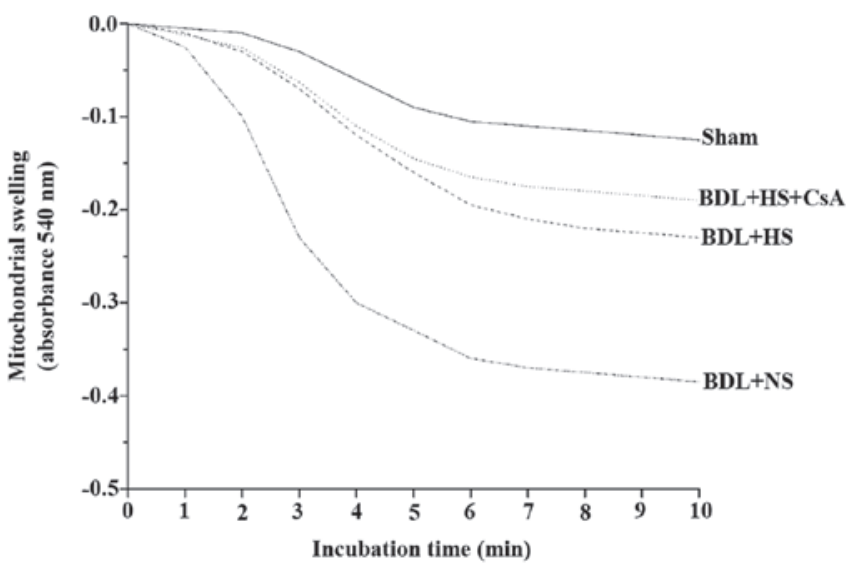

Figure 3. Mitochondrial swelling in the liver of BDL mice. Mitochondrial swelling was monitored by decreases in absorbance at $540 \mathrm{~nm}$ following the addition of $100 \mu \mathrm{M} \mathrm{CaCl}_{2}$. The dotted lines indicate the incubation of mitochondria from BDL mice treated with NS, HS or CsA. The results are representative of four experiments. HS, hydrogen-rich saline; BDL, bile duct ligation; NS, normal saline; CsA, cyclosporin A. 


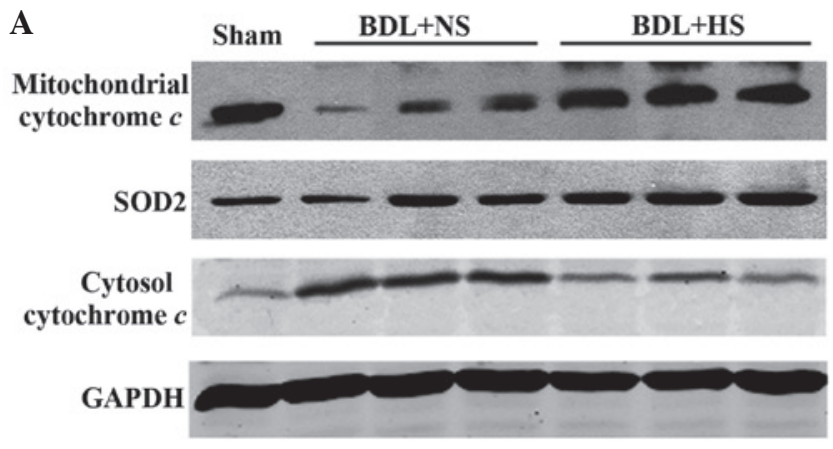

C

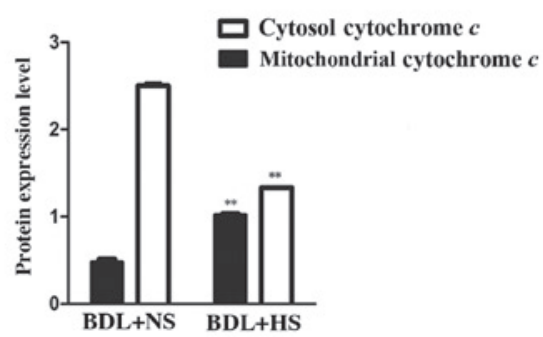

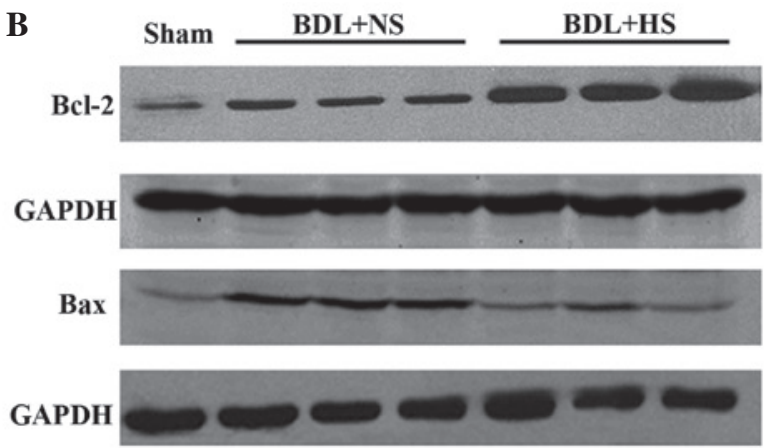

D

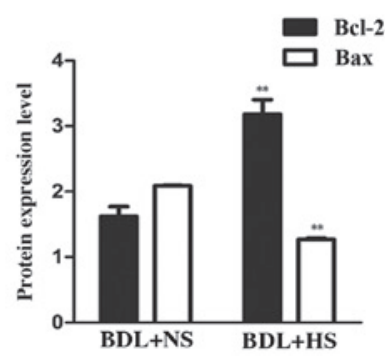

Figure 4. Western blot analysis of cytochrome $c, \mathrm{Bcl}-2$ and Bax protein expression levels in BDL mice. (A) Mitochondrial and cytosolic lysates were subjected to western blotting using cytochrome $c$-specific antibodies. Treatment with HS significantly suppressed the release of cytochrome $c$ from the mitochondria into the cytosol. Images are representative of four independent experiments. SOD2 and GAPDH were used as the mitochondrial and cytosolic loading controls, respectively. (B) Liver lysates were subjected to western blot analysis using Bcl-2 and Bax-specific antibodies. The protein expression levels of Bax were increased, whereas Bcl-2 expression was decreased in livers from HS-treated mice compared with those obtained from NS-treated mice. Images are representative of four independent experiments. GAPDH was used as a loading control for normalization. (C and D) Western blotting quantification. Data are presented as the mean \pm standard deviation. ${ }^{* *} \mathrm{P}<0.01$ vs. the BDL + NS group. HS, hydrogen-rich saline; BDL, bile duct ligation; NS, normal saline; SOD2, manganese superoxide dismutase; GAPDH, glyceraldehyde 3-phosphate dehydrogenase; Bcl-2, B-cell lymphoma 2; Bax, Bcl-2-associated X protein.

Bioluminescent Assay kit (Sigma-Aldrich Canada, Oakville, ON, Canada), according to the manufacturer's protocol.

Determination of mitochondrial respiratory function. Mitochondrial respiratory function was determined using the Clark Oxygen Electrode system (Oxygraph ${ }^{\mathrm{TM}}$, Hansatech Instruments, Ltd., King's Lynn, UK), according to previously described methods (21). The mitochondrial respiratory control ratio (RCR) and adenosine diphosphate (ADP) to oxygen ratio $(\mathrm{ADP} / \mathrm{O})$ can reflect mitochondrial respiratory function and integrity of the respiratory chain. Mitochondrial RCR is the ratio of state 3 and 4 respiration rates. ADP/O is the ratio of ADP and state 3 oxygen consumption. State 3 is the respiration rate after the addition of $1 \mathrm{mM}$ ADP; whereas state 4 is the oxygen consumption rate after the complete phosphorylation of ADP.

Statistical analysis. All experiments were performed in triplicate and statistical analyses were conducted using SPSS 22.0 software (IBM SPSS, Armonk, NY, USA). Data are presented as the mean \pm standard deviation. Statistical analyses were performed using one-way analysis of variance (ANOVA) for the comparison of three groups. When ANOVA exhibited significant differences, pairwise comparisons between means were tested by Student-Newman-Keuls post-hoc test. $\mathrm{P}<0.05$ was considered to indicate a statistically significant difference.

\section{Results}

HS prevents mitochondrial oxidative stress and increases antioxidant activities in BDL mice. The levels of mMDA were significantly increased $(99.6 \%)$ in the livers of BDL mice compared with in the sham-operated mice. Conversely, treatment with HS markedly prevented the elevation of lipid peroxidation in mitochondria (Fig. 1A; $\mathrm{P}=0.006$ ). The potential antioxidative properties of HS were determined by measuring the levels of mGSH and mGSSG. In NS-treated BDL mice, mGSH levels were reduced to $58.9 \%$ of normal levels and mGSSG levels were increased by 1.276-fold; however, HS significantly attenuated the altered $\mathrm{mGSH}$ and $\mathrm{mGSSG}$ levels (Fig. $1 \mathrm{~B}$ and $\mathrm{C} ; \mathrm{P}=0.005$ and $\mathrm{P}=0.004$, respectively). The results also indicated that an increase in mitochondrial oxidative stress was accompanied by a significant decrease in the activities of the antioxidant enzymes SOD, CAT and Gpx in the hepatic mitochondria of BDL mice, whereas HS treatment markedly increased antioxidant activities in BDL mice (Fig. 1D-F; $\mathrm{P}=0.006, \mathrm{P}=0.005$ and $\mathrm{P}=0.009$, respectively).

HS decreases apoptosis in hepatocytes from BDL mice. HS markedly reduced the number of TUNEL-positive cells in the liver compared with the NS-treated group (Fig. 2A), thus suggesting that HS is able to inhibit BDL-induced hepatocyte apoptosis. To further confirm that HS affected hepatocyte apoptosis, flow cytometric analysis was conducted using Annexin V-FITC and PI staining, in order to discriminate between apoptotic and necrotic cells. In BDL mice, treatment with HS induced significantly lower levels of apoptosis $(39.8 \%)$ compared with in the NS-treated group (52.5\%) (Fig. 2B and C; both $\mathrm{P}=0.008$ ).

HS inhibits caspase activities in the livers of BDL mice. Since caspase activation has a key role in apoptotic cell death, the 

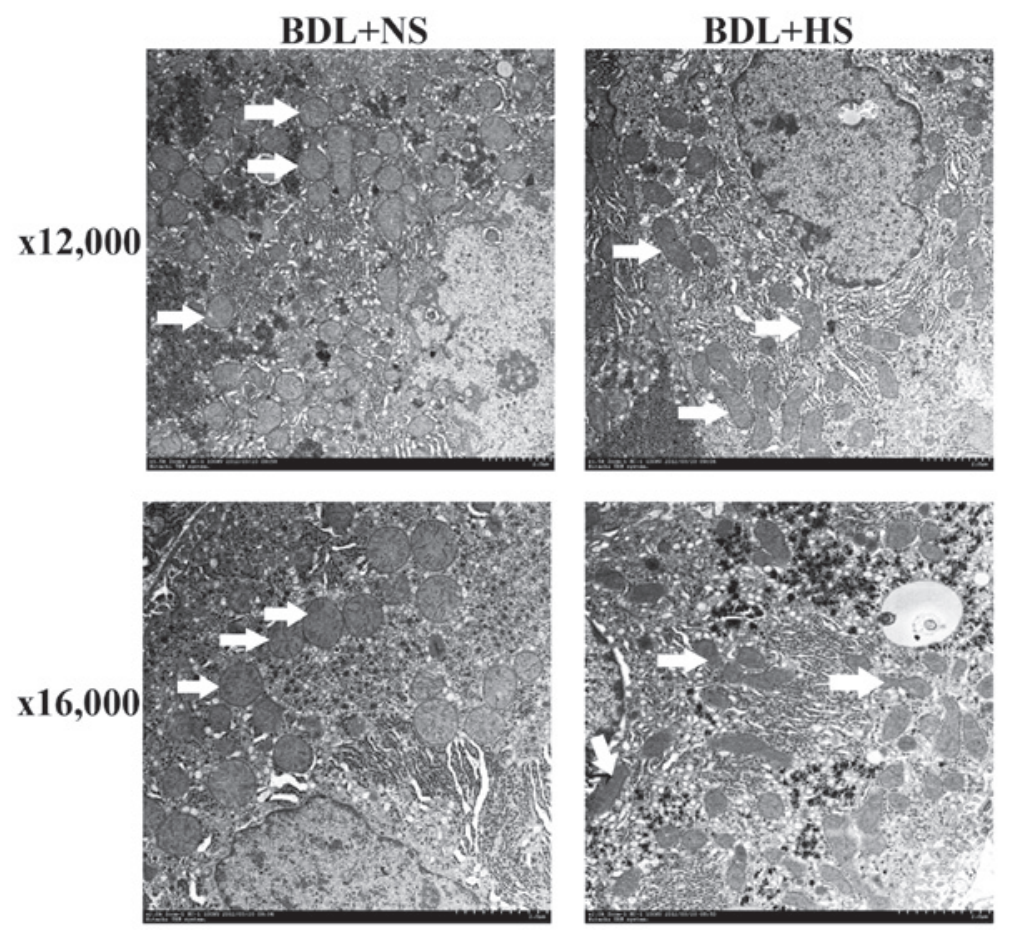

Figure 5. Ultrastructural analysis of hepatocyte mitochondria using TEM. In addition to the observed swelling, TEM studies clearly indicated impaired mitochondria, with an absent double membrane; distorted cristae, which were far fewer than in controls; and an appreciable reduction in electron-dense granules in the intramitochondrial matrix. All of these ultrastructural modifications were markedly alleviated following treatment with HS. Arrows indicate mitochondria. TEM, transmission electron microscopy; BDL, bile duct ligation; NS, normal saline; HS, hydrogen-rich saline.

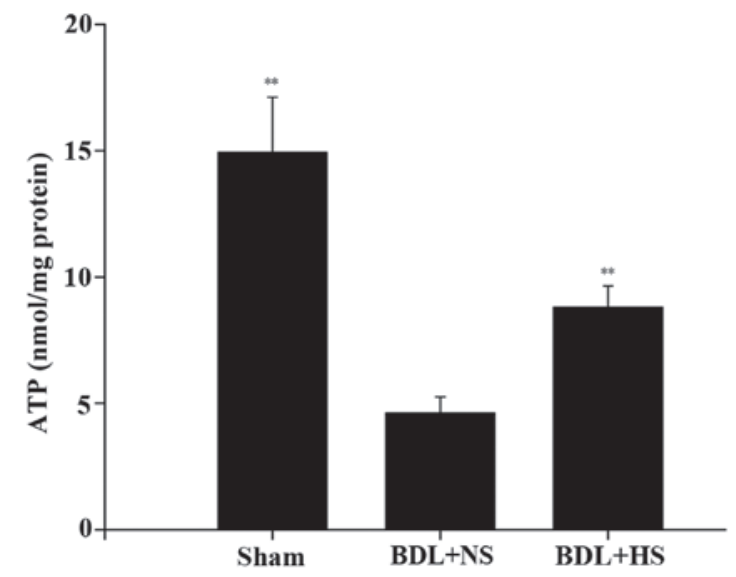

Figure 6. ATP levels in the hepatocytes of BDL mice. ATP content was measured in isolated mitochondria using a luciferase-based assay. Data are presented as the mean \pm standard deviation. ${ }^{* *} \mathrm{P}<0.01$, vs. the BDL group. ATP, adenosine triphosphate; HS, hydrogen-rich saline; BDL, bile duct ligation.

present study further investigated whether caspase activities could be altered following treatment with HS. BDL triggered a significant increase in hepatic caspase 3,8 and 9 activities, whereas HS administration significantly reduced caspase activities (Fig. 2D; $\mathrm{P}=0.007, \mathrm{P}=0.008$ and 0.007 , respectively). These findings were consistent with the results of the apoptosis analysis.

HS inhibits mitochondrial swelling. Mitochondria from the sham-operated mice tolerated $\mathrm{Ca}^{2+}$ at a concentration of $100 \mu \mathrm{M}$ without undergoing MPT, as assessed by a mito- chondrial swelling assay (22). Conversely, mitochondria from the NS-treated BDL mice were much more sensitive to MPT induction. A large-amplitude swelling was observed in mitochondria isolated from NS-treated BDL mice compared with in the mitochondria from sham-operated mice. In addition, treatment with HS prevented BDL-induced mitochondrial swelling (Fig. 3).

$H S$ inhibits mitochondrial cytochrome c release in the liver of BDL mice. Western blot analysis (Fig. 4) indicated that NS-treated BDL mice exhibited a significant reduction in the protein expression levels of mitochondrial cytochrome $c$, which was accompanied by a release into the cytoplasm, as reflected by an increase in cytosolic cytochrome $c$ expression levels (Fig. 4A and C; $\mathrm{P}=0.008$ ). Treatment with HS significantly inhibited the release of cytochrome $c$ from the mitochondria to the cytoplasm (Fig. 4A and $\mathrm{C} ; \mathrm{P}=0.006$ ).

$H S$ prevents alterations in mitochondrial Bcl-2 family protein expression in the liver of BDL mice. Pro-apoptotic and anti-apoptotic members of the Bcl-2 protein family have critical roles in regulating the MPT and cytochrome $c$ release (23). Therefore, the present study evaluated the mitochondrial expression levels of Bcl-2 and Bax in BDL livers. Western blotting demonstrated a significant increase in Bax protein expression, and a concomitant reduction in Bcl-2 protein expression in the mitochondria of BDL mice (Fig. 4B and $\mathrm{D} ; \mathrm{P}=0.006)$. These BDL-induced effects were prevented by HS treatment (Fig. 4B and D; P=0.008).

$H S$ protects mitochondrial ultrastructure in BDL mice. TEM analysis of liver tissue indicated that mitochondrial 

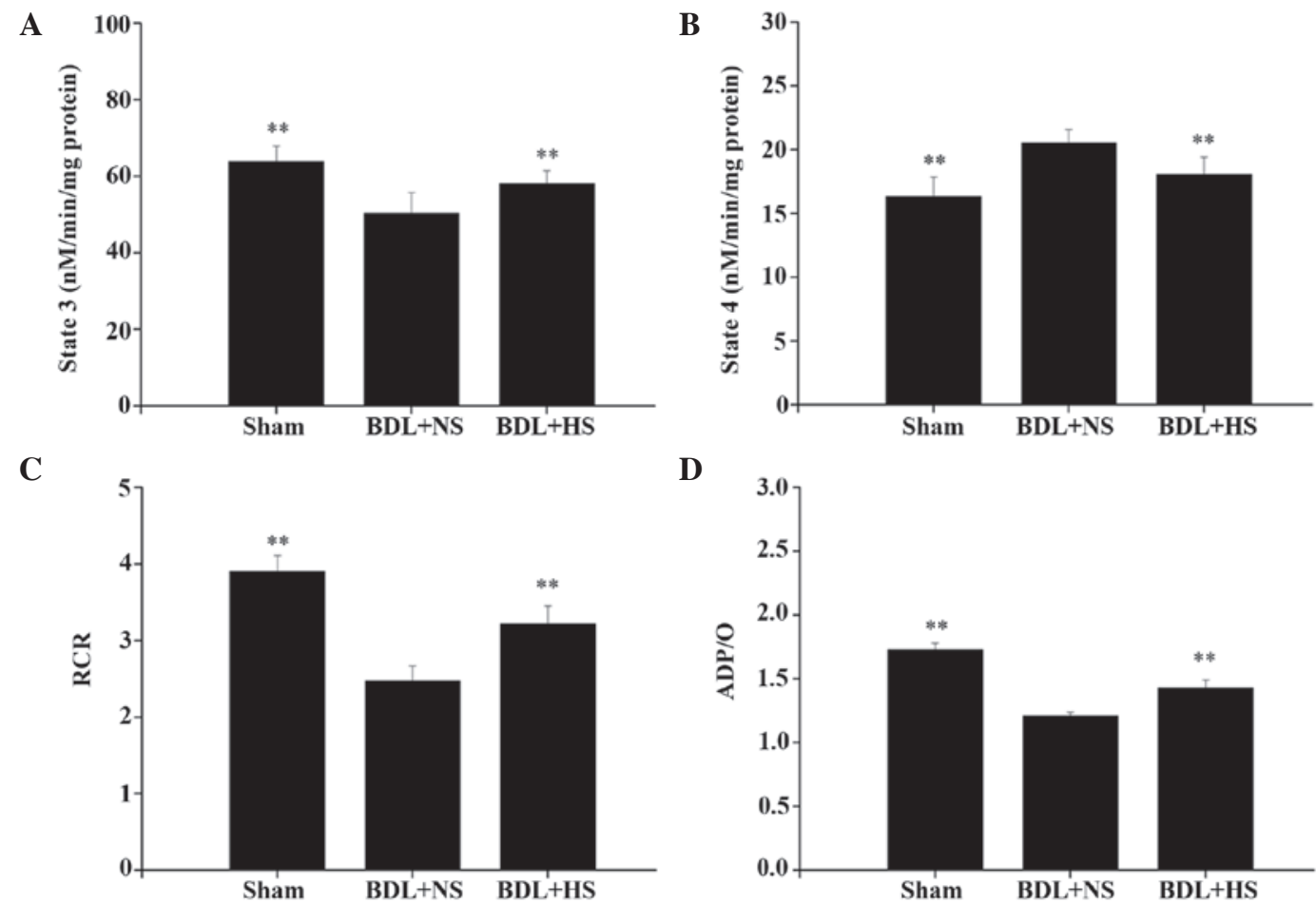

Figure 7. Phosphorylation (State 3) and resting (State 4) respiratory rates in liver mitochondria isolated from sham-operated and BDL mice, treated with NS or HS. (A) State 3 respiration rates. (B) State 4 respiration rates. (C) Mitochondrial RCR and (D) ADP/O ratios. Results are presented as the mean \pm standard deviation $(\mathrm{n}=20)$. State 3 and State 4 respiration rates are expressed as $\mathrm{nM}$ oxygen $/ \mathrm{min} / \mathrm{mg}$ protein. The mitochondrial RCR is a ratio of state 3 and state 4 . ${ }^{* *} \mathrm{P}<0.01$ vs. the BDL + NS group. HS, hydrogen-rich saline; BDL, bile duct ligation; NS, normal saline; RCR, respiratory control ratio; ADP/O, adenosine diphosphate to oxygen.

ultrastructural alterations occurred in the hepatocytes of NS-treated BDL mice in the present study. In addition to the observed swelling, TEM analysis clearly showed impaired mitochondria, with an absent double membrane; distorted cristae, which were far fewer than in the sham mice; and an appreciable reduction in electron-dense granules in the intramitochondrial matrix. All of these ultrastructural modifications were markedly alleviated following treatment with HS (Fig. 5).

HS prevents the depletion of ATP levels in hepatocytes of $B D L$ mice. The present study examined the effects of HS on ATP levels, which is a sensitive parameter of mitochondrial function. ATP levels were decreased $(67.1 \%)$ in BDL mice compared with in the sham-operated mice. Conversely, treatment with HS significantly increased ATP levels in the hepatocytes of BDL mice (Fig. 6; $\mathrm{P}=0.007$ ).

HS improves the BDL-induced decline in mitochondrial respiratory function. BDL induced a significant reduction in RCR (31.3\%) compared with in the sham-operated mice $(\mathrm{P}<0.01)$. This decrease in RCR could be principally attributed to the observed significant decrease $(18.5 \%)$ of State 3 (Fig. 7A; $\mathrm{P}=0.009$ ), as opposed to the $19.3 \%$ increase of State 4 (Fig. 7B; $\mathrm{P}=0.009$ ). HS promoted a significant increase in RCR compared with the NS group (Fig. 7C; $\mathrm{P}=0.08$ ). This protection was principally related to an increase of State 3 and a decrease of State 4 (Fig. 7A and B). ADP/O was also significantly increased (17.4\%) following HS treatment (Fig. 7D; $\mathrm{P}=0.009$ ).

\section{Discussion}

OJ induces ROS generation and tumor necrosis factor- $\alpha$ expression in the liver, both of which can induce depolarization of the mitochondrial membrane and eventually initiate apoptosis (24). Our previous study demonstrated that HS was able to ameliorate BDL-induced liver injury by reducing oxidative stress and inflammatory cascades in liver tissue (14). Theoretically, $\mathrm{H}_{2}$ is highly diffusible and could potentially reach mitochondria, which are targets of excessive ROS and central mediators of apoptosis $(25,26)$. Therefore, the aim of the present study was to evaluate whether HS, a selective antioxidant, has the capacity to reduce mitochondrial oxidative stress, and thus protect against mitochondrial dysfunction and inhibit mitochondrial apoptosis, in order to prevent BDL-induced liver injury.

Mitochondria are the major source and target of excessive ROS (26). Mitochondrial GSH scavenges free oxygen radicals that are generated by the mitochondrial respiratory chain (27) and antioxidant enzymes, including SOD, CAT and Gpx, work synergistically to cope with oxidative stress. Accumulated exposure to ROS leads to an oxidative stress burden in the mitochondria, which can induce an apparent increase in ROS generation by the electron transfer chain and suppress the mitochondrial antioxidant system (28). In the present study, mMDA was significantly increased by BDL. In addition, OJ was revealed to impair the activities of mSOD, mCAT and mGpx by $36.1,53.8$ and $32.2 \%$, respectively, and mGSH was also depleted by BDL-induced oxidative stress. Treatment with HS markedly decreased mMDA levels, increased mGSH 
levels, and elevated the activities of mSOD, mCAT and mGpx, thus suggesting that $\mathrm{H}_{2}$ may reduce mitochondrial lipid peroxidation, boost antioxidant capacity and maintain the mitochondrial redox balance.

Mitochondrial oxidative stress triggers the opening of the MPT pore, and the simultaneous collapse of the mitochondrial membrane potential (MMP) (29-31). MPT further increases the permeability of the mitochondrial outer membrane and induces mitochondrial swelling $(30,31)$. Furthermore, cytochrome $c$, a mitochondrial intermembrane protein, is released into the cytosol via specific channels, including the MPT pore and Bax channel, and may further activate the downstream caspase pathway to induce irreversible apoptosis (31).

The present study used $100 \mu \mathrm{M} \mathrm{CaCl}_{2}$ as an MPT inducer, and demonstrated that HS-treated BDL mice exhibited a decrease in mitochondrial swelling compared with the NS-treated mice, thus indicating that HS inhibits the opening of the MPT pore and prevents the onset of MPT. Similar results were obtained following an intraperitoneal injection of CsA for MPT protection. CsA, which is a specific MPT inhibitor, was able to significantly protect against BDL-induced mitochondrial swelling. The combination of CsA and HS offered more efficient protection than HS or CsA alone against BDL-induced mitochondrial swelling. Therefore, it may be hypothesized that part of the mechanism involved in HS-induced protection is via inhibition of MPT. The present study also detected leakage of cytochrome $c$ into the cytosol in BDL mice, which is correlated with MPT pore opening. Conversely, treatment with HS prevented the release of cytochrome $c$ into the cytosol.

Mitochondria are the central control point of apoptosis (32). OJ induces apoptotic cell death, which is associated with mitochondrial oxidative stress, MMP alteration and cytochrome $c$ release $(33,34)$. In the present study, BDL resulted in the accumulation of TUNEL-positive and Annexin-V-positive cells. The TUNEL-positive and Annexin-V-positive cells were markedly decreased in the HS-treated group, thus suggesting that HS may provide hepatic protection via its anti-apoptotic activity.

Members of the Bcl-2 family are key players in the mitochondrial intrinsic pathway of apoptosis (35). The Bcl-2 family consists of pro- and anti-apoptotic proteins that work together to mediate mitochondrial integrity and maintain a dynamic balance between cell survival and cell death. Bax integrates with the permeability transition pore complex and forms specific proteolipid channels in the outer membrane, in order to promote cytochrome $c$ release, whereas $\mathrm{Bcl}-2$ directly binds to Bax to inhibit formation of the proteolipid pore (36-38). In the present study, BDL increased the expression of Bax and decreased the expression of Bcl-2 in the liver, whereas treatment with HS markedly attenuated BDL-induced elevation of Bax expression and reduction of Bcl-2 expression. These data suggested that HS may markedly suppress downstream apoptotic events by modulating the expression levels of Bcl-2 family members.

The caspase family consists of cysteine proteases that can cleave target proteins at specific aspartate residues. Previous studies have reported that caspases have important roles in the initiation, regulation and execution of apoptosis of hepatocytes in response to BDL-induced injury (38-40). One of the main consequences following mitochondrial cytochrome $c$ release is the activation of caspase 3 through the apoptosome, which consists of cytochrome $c$, apoptotic protease activating factor-1 and procaspase 9 (9). Caspases 3 and 9 are downstream effectors in the caspase-dependent intrinsic apoptosis pathway, whereas caspase 8 is an essential part of the extrinsic pathway. The present study demonstrated that activation of caspases 3 , 8 and 9 was significantly suppressed in HS-treated mice, thus indicating that HS may inhibit BDL-induced apoptosis via both intrinsic and extrinsic pathways.

The results of the present study demonstrated that HS was able to protect mitochondrial respiratory function and attenuate the depletion of mitochondrial ATP in BDL mice. In addition, HS was shown to attenuate mitochondrial ultrastructural injury, as evidenced by TEM observations. These results strongly suggested that HS may effectively prevent BDL-induced mitochondrial dysfunction.

In conclusion, the present study provides some of the first evidence to suggest that HS is able to ameliorate BDL-induced acute liver damage by reducing mitochondrial ROS production, protecting against mitochondrial dysfunction, and inhibiting mitochondria-mediated apoptosis. These findings provide evidence regarding the possible mechanisms underlying the protective role of HS. Furthermore, $\mathrm{H}_{2}$ treatment appears to decrease liver fibrosis, cirrhosis, and overall mortality during long-term cholestasis (unpublished data). Since mitochondria are known to be involved in numerous signaling pathways and there are limited data from clinical trials involving HS treatment, it remains to be elucidated whether the regulatory effects of HS on gene pathways associated with apoptosis and oxidative stress have direct clinical value.

\section{Acknowledgements}

The present study was supported by a grant from the National Natural Science Foundation of China (grant no. 31170926).

\section{References}

1. Poupon R, Chazouillères $\mathrm{O}$ and Poupon RE: Chronic cholestatic diseases. J Hepatol 32 (Suppl 1): 129-140, 2000.

2. Greig JD, Krukowski ZH and Matheson NA: Surgical morbidity and mortality in one hundred and twenty-nine patients with obstructive jaundice. Br J Surg 75: 216-219, 1988.

3. Vendemiale G, Grattagliano I, Lupo L, Memeo V and Altomare E: Hepatic oxidative alterations in patients with extra-hepatic cholestasis. Effect of surgical drainage. J Hepatol 37: 601-605, 2002.

4. Assimakopoulos SF, Vagianos CE, Zervoudakis G, Filos KS, Georgiou C, Nikolopoulou V and Scopa CD: Gut regulatory peptides bombesin and neurotensin reduce hepatic oxidative stress and histological alterations in bile duct ligated rats. Regul Pept 120: 185-193, 2004.

5. Liu TZ, Lee KT, Chern CL, Cheng JT, Stern A and Tsai LY: Free radical-triggered hepatic injury of experimental obstructive jaundice of rats involves overproduction of proinflammatory cytokines and enhanced activation of nuclear factor kappaB. Ann Clin Lab Sci 31: 383-390, 2001.

6. Kantrow SP, Tatro LG and Piantadosi CA: Oxidative stress and adenine nucleotide control of mitochondrial permeability transition. Free Radic Biol Med 28: 251-260, 2000.

7. Kim JS, He L, Qian T and Lemasters JJ: Role of the mitochondrial permeability transition in apoptotic and necrotic death after ischemia/reperfusion injury to hepatocytes. Curr Mol Med 3: 527-535, 2003.

8. Miyoshi H, Rust C, Roberts PJ, Burgart LJ and Gores GJ: Hepatocyte apoptosis after bile duct ligation in the mouse involves Fas. Gastroenterology 117: 669-677, 1999. 
9. Canbay A, Feldstein A, Baskin-Bey E, Bronk SF and Gores GJ: The caspase inhibitor IDN-6556 attenuates hepatic injury and fibrosis in the bile duct ligated mouse. J Pharmacol Exp Ther 308 : 1191-1196, 2004

10. Wang L, Hartmann P, Haimerl M, Bathena SP, Sjöwall C, Almer S, Alnouti Y, Hofmann AF and Schnabl B: Nod2 deficiency protects mice from cholestatic liver disease by increasing renal excretion of bile acids. J Hepatol 60: 1259-1267, 2014

11. Ohsawa I, Ishikawa M, Takahashi K, Watanabe M, Nishimaki K Yamagata K, Katsura K, Katayama Y, Asoh S and Ohta S: Hydrogen acts as a therapeutic antioxidant by selectively reducing cytotoxic oxygen radicals. Nat Med 13: 688-694, 2007.

12. Zheng X, Mao Y, Cai J, Li Y, Liu W, Sun P, Zhang JH, Sun X and Yuan H: Hydrogen-rich saline protects against intestinal ischemia/reperfusion injury in rats. Free Radic Res 43 478-484, 2009.

13. Kajiya M, Silva MJ, Sato K, Ouhara K and Kawai T: Hydrogen mediates suppression of colon inflammation induced by dextran sodium sulfate. Biochem Biophys Res Commun 386: 11-15, 2009.

14. Liu Q, Shen WF, Sun HY, Fan DF, Nakao A, Cai JM, Yan G Zhou WP, Shen RX, Yang JM and Sun XJ: Hydrogen-rich saline protects against liver injury in rats with obstructive jaundice. Liver Int 30: 958-968, 2010.

15. Cai J, Kang Z, Liu K, Liu W, Li R, Zhang JH, Luo X and Sun X: Neuroprotective effects of hydrogen saline in neonatal hypoxia-ischemia rat model. Brain Res 1256: 129-137, 2009.

16. Zhuge J and Cederbaum AI: Serum deprivation-induced HepG2 cell death is potentiated by CYP2E1. Free Radic Biol Med 40: 63-74, 2006

17. Zhuge J and Cederbaum AI: Increased toxicity by transforming growth factor-beta 1 in liver cells overexpressing CYP2E1. Free Radic Biol Med 41: 1100-1112, 2006.

18. Sherwood SW and Schimke RT: Cell cycle analysis of apoptosis using flow cytometry. Methods Cell Biol 46: 77-97, 1995.

19. Wu D and Cederbaum A: Cytochrome P4502E1 sensitizes to tumor necrosis factor alpha-induced liver injury through activation of mitogen-activated protein kinases in mice. Hepatology 47: 1005-1017, 2008.

20. Rickmann M, Vaquero EC, Malagelada JR and Molero X: Tocotrienols induce apoptosis and autophagy in rat pancreatic stellate cells through the mitochondrial death pathway. Gastroenterology 132: 2518-2532, 2007.

21. Yang L and $\mathrm{Yu} \mathrm{T}$ : Prolonged donor heart preservation with pinacidil: The role of mitochondria and the mitochondrial adenosine triphosphate-sensitive potassium channel. J Thorac Cardiovasc Surg 139: 1057-1063, 2010.

22. Gogvadze V, Orrenius S and Zhivotovsky B: Analysis of mitochondrial dysfunction during cell death. Curr Protoc Cell Biol Chapter 18: Unit 18.5, 2003

23. Youle RJ and Strasser A: The BCL-2 protein family: Opposing activities that mediate cell death. Nat Rev Mol Cell Biol 9: 47-59, 2008.

24. Palmeira CM and Rolo AP: Mitochondrially-mediated toxicity of bile acids. Toxicology 203: 1-15, 2004.

25. Sun Q, Kang Z, Cai J, Liu W, Liu Y, Zhang JH, Denoble PJ, Tao $\mathrm{H}$ and Sun $\mathrm{X}$ : Hydrogen-rich saline protects myocardium against ischemia/reperfusion injury in rats. Exp Biol Med (Maywood) 234: 1212-1219, 2009.
26. Mantena SK, King AL, Andringa KK, Eccleston HB and Bailey SM: Mitochondrial dysfunction and oxidative stress in the pathogenesis of alcohol- and obesity-induced fatty liver diseases. Free Radic Biol Med 44: 1259-1272, 2008.

27. Cederbaum AI, Lu Y and Wu D: Role of oxidative stress in alcohol-induced liver injury. Arch Toxicol 83: 519-548, 2009.

28. Zorov DB, Juhaszova M and Sollott SJ: Mitochondrial ROS-induced ROS release: An update and review. Biochim Biophys Acta 1757: 509-517, 2006.

29. Zorov DB, Filburn CR, Klotz LO, Zweier JL and Sollott SJ: Reactive oxygen species (ROS)-induced ROS release: A new phenomenon accompanying induction of the mitochondrial permeability transition in cardiac myocytes. J Exp Med 192: 1001-1014, 2000

30. Petronilli V, Costantini P, Scorrano L, Colonna R, Passamonti S and Bernardi P: The voltage sensor of the mitochondrial permeability transition pore is tuned by the oxidation-reduction state of vicinal thiols. Increase of the gating potential by oxidants and its reversal by reducing agents. J Biol Chem 269: 16638-16642, 1994.

31. Kim JS, He L and Lemasters JJ: Mitochondrial permeability transition: A common pathway to necrosis and apoptosis. Biochem Biophys Res Commun 304: 463-470, 2003.

32. Adams JM and Cory S: The Bcl-2 protein family: Arbiters of cell survival. Science 281: 1322-1326, 1998.

33. Malhi H, Gores GJ and Lemasters JJ: Apoptosis and necrosis in the liver: A tale of two deaths? Hepatology 43 (2 Suppl 1): S31-S44, 2006

34. Wei MC, Zong WX, Cheng EH, Lindsten T, Panoutsakopoulou V, Ross AJ, Roth KA, MacGregor GR, Thompson CB and Korsmeyer SJ: Proapoptotic BAX and BAK: A requisite gateway to mitochondrial dysfunction and death. Science 292: 727-730, 2001.

35. Chaudhary K, Liedtke C, Wertenbruch S, Trautwein C and Streetz KL: Caspase 8 differentially controls hepatocytes and non-parenchymal liver cells during chronic cholestatic liver injury in mice. J Hepatol 59: 1292-1298, 2013.

36. Tiao MM, Lin TK, Liou CW, Wang PW, Chen JB, Kuo FY, Huang CC, Chou YM and Chuang JH: Early transcriptional deregulation of hepatic mitochondrial biogenesis and its consequent effects on murine cholestatic liver injury. Apoptosis 14: 890-899, 2009.

37. Kahraman A, Barreyro FJ, Bronk SF, Werneburg NW, Mott JL, Akazawa Y, Masuoka HC, Howe CL and Gores GJ: TRAIL mediates liver injury by the innate immune system in the bile duct-ligated mouse. Hepatology 47: 1317-1330, 2008.

38. Green DR and Reed JC: Mitochondria and apoptosis. Science 281: 1309-1312, 1998

39. Yoon JH and Gores GJ: Death receptor-mediated apoptosis and the liver. J Hepatol 37: 400-410, 2002

40. Marin JJ, Hernandez A, Revuelta IE, Gonzalez-Sanchez E, Gonzalez-Buitrago JM and Perez MJ: Mitochondrial genome depletion in human liver cells abolishes bile acid-induced apoptosis: Role of the Akt/mTOR survival pathway and Bcl-2 family proteins. Free Radic Biol Med 61: 218-228, 2013. 